Bangladesh J. Plant Taxon. 12(2): 85-96, 2005 (December)

\title{
A CHECKLIST OF ANGIOSPERMIC FLORA OF LALMAI HILLS, COMILLA, BANGLADESH
}

\author{
Md. Munir Hossain, Md. Abul Hassan and Mohammad Zashim Uddin \\ Department of Botany, University of Dhaka, Dhaka-1000, Bangladesh
}

Key words: Preliminary checklist, angiospermic flora, Lalmai hills, Comilla, Bangladesh

\begin{abstract}
The paper includes a checklist containing a total of 151 angiospermic species (127 dicot and 24 monocot) growing in the Lalmai hills, Comilla, Bangladesh, which has been prepared after the survey of the area during the years 2001 and 2002.
\end{abstract}

\section{Introduction}

The Lalmai hills are situated in the district of Comilla, about $8 \mathrm{~km}$ to the west of Comilla town. They lie on $23^{\circ}-21^{\prime} \mathrm{N}$ latitude and $91^{\circ}-09^{\prime} \mathrm{E}$ longitude. The hills are commonly known as Mainamati - Lalmai range. The range includes 50 hillocks (Hossen and Dewan 2004). The range extends from north to south for a distance of $17.71 \mathrm{~km}$ from Mainamati to Datya Dighi and is about $40.25 \mathrm{~km}$ in circumference.

The area of investigation consists of several hills of different heights and a few valleys. The hills attain a height of $45 \mathrm{~m}$ at some places, but the average elevation is $12 \mathrm{~m}$ above the plains. The highest peaks are Kalir Bazar and Chand Mura, and there are small springs near these peaks and Bijaynagar. The hills are bounded by the faults on the western and eastern sides. The faults scrap on the east is up-thrown to the west and considerably dissected by drainage channels. On the west there are two parallel faults about $1.5 \mathrm{~km}$ apart of which both are up-thrown on the east. The inner fault forms a welldefined valley, while the outer (westernmost) fault has a surface throw in excess of $30 \mathrm{~m}$ at several places. Structurally this range is a horst, tilted to the east.

The hills are flat with steep and strongly gullied sides, mainly consisting of unconsolidated sands with a clay capping. The old piedmont apron comprises a narrow fringe of foothills along with the Chittagong-Tripura hills in India with a gently flooding relief. It also comprises the foothills of Lalmai hills, mainly consisting of unconsolidated finer textured and moderately well-drained soils derived from weathered Tertiary or Pleistocene unconsolidated sands and soils. These strongly acid soils occur on gently rolling areas of the Lalmai hills. They have yellowish-brown topsoil overlying a strong brown to yellowish-red structured clay subsoil. Texture of the surface layer varies from sandy-clay-loam to clay-loam. These soils are moderately well-drained with medium to rapid runoff and medium internal drainage, which are droughty in the dry season. The forest enjoys a tropical climate characterized by a period of high precipitation from May to October and six months of relatively dry period from November to April.

The Lalmai supports a moist deciduous Sal forest. It supports a large number of plant species that would contribute to the forest economy of Bangladesh. But the forest of 
Lalmai hills area does not have any published flora or manual. At the same time this area is vulnerable and needs to be documented and protected immediately.

\section{Materials and Methods}

The present work is based mainly on the fresh materials collected by the authors from the study area through repeated field trips during the year 2001-2002. These are supplemented by the herbarium specimens examined at the Dhaka University Herbarium and Bangladesh National Herbarium. The identification of the specimens was confirmed with the help of Hooker (1872-1897), Prain (1903, 1903a), Brandis (1906), Kanjilal et al. (1934, 1938, 1939, 1940), Khan $(1977,1984,1985)$, Deb (1981, 1983) and Matthew (1999, 1999a, 1999b). The families have been arranged according to Cronquist (1981). The genera and species under each family are arranged in alphabetical order. The checklist includes wild, naturalized and planted species.

\section{Results}

A total of 149 species under 127 genera and 49 families have been identified and recorded in this paper. Magnoliopsida is represented by 43 families, 108 genera and 127 species while Liliopsida is represented by 6 families, 19 genera and 22 species. In Magnoliopsida, the Fabaceae appears to be the largest family having 9 genera and 12 species. In Liliopsida, the Poaceae appears to be the largest family having 8 genera and 11 species.

\section{MAGNOLIOPSIDA (DICOTS)}

\section{Magnoliaceae}

Michelia champaca L., Sp. Pl.: 536(1753). Local name: Champa. A mediumsized tree.

\section{Ulmaceae}

Trema orientalis (L.) Bl., Mus. Bot. Lugd. Bot. 2: 62 (1856). Celtis orientalis L., Sp. Pl.: 1044(1753). A small to medium-sized tree.

\section{Moraceae}

Artocarpus chama Buch.-Ham. ex Wall. Cat.: 4657(1814). Artocarpus chaplasha Roxb., Fl. Ind. 3: 525(1832). Local name: Chamul. A lofty tree.

A. heterophyllus Lamk., Meth. B. 3: 209(1789). Local name: Kanthal. An evergreen tree.

Ficus benghalensis L., Sp. Pl.: 1059(1753). Local name: Bot. A large spreading tree.

F. hispida L. f., Suppl. Pl.: 442(1781). Local name: Dumur. A low tree.

F. religiosa L., Sp. Pl.: 1059(1753). Local name: Ashathwa. A large tree.

Streblus asper Lour., Fl. Cochin. 2: 615(1790). Local name: Sheora. A bushy tree.

\section{Amaranthaceae}

Achyranthes aspera L., Sp. Pl.: 204(1753). Local name: Apang. A perennial herb. 
Aerva lanata (L.) Juss. ex Schult., Syst. Veg. 15(5): 564(1819). Achyranthes lanata L., Sp. Pl. 1: 204(1753). Local name: Chaya. An erect herb.

Amaranthus viridis L., Sp. Pl. ed. 2: 1405(1763). Local name: Notey Shak. A slender herb.

Cyathula prostrata (L.) Blume, Bijdr.: 549(1825). Achyranthes prostrata L., Sp. Pl. ed. 2: 296(1762). An annual herb.

\section{Dipterocarpaceae}

Dipterocarpus turbinatus Gaertn. f., De Fruct. 3: 51, t. 188, f. 1(1805). Local name: Telia Garjon. A lofty evergreen tree.

Shorea robusta Roxb. ex Gaertn. f., De Fruct. 3: 48. t. 186(1805). Local name: Sal, Gajari. A tall deciduous tree.

\section{Elaeocarpaceae}

Elaeocarpus robustus Roxb. [Hort. Beng.: 42(1814). nom. nud.], Fl. Ind. 2: 597(1824). Local name: Jalpai. A small tree.

\section{Tiliaceae}

Grewia serrulata DC. Prodr. 1: 510(1824). Local name: Pichandi. A shrub. Microcos paniculata L., Sp. Pl. 1: 514(1753). Local name: Asar. A shrub to small tree.

Triumfetta rhomboidea Jacq. [Enum. Pl. Carib.: 22(1762) nomen], Select. Strip. Am.: 147. t. 90(1763). Local name: Banokra. An undershrub.

\section{Bombacaceae}

Bombax ceiba L., Sp. Pl.: 511(1753). Local name: Shimul Tula. A large tree with buttress base.

\section{Malvaceae}

Hibiscus sabdariffa L., Sp. Pl.: 695(1753). Local name: Mesta. A shrub.

Sida acuta Burm. f., Fl. Ind.: 147(1768). Local name: Kureta. A herb.

S. cordata (Burm. f.) Borss. in Blumea 14(1): 182(1966). Melochia cordata Burm. f., Fl. Ind.: 143(1768). Local name: Junka. An annual herb.

S. mysorensis Wt. \& Arn., Prodr.: 59(1834). An annual herb.

S. rhombifolia L., Sp. Pl.: 684(1753). An undershrub.

Urena lobata L., Sp. Pl.: 692(1753). Local name: Jaruga gota. An undershrub.

\section{Lecythidaceae}

Careya arborea Roxb., Pl. Corom. 3: 14. t. 218(1811). Local name: Kumbi. A low tree.

\section{Flacourtiaceae}

Flacourtia indica (Burm.f.) Merril, Interpr. Rumph. Herb. Amb.: 377(1917). Gmelina 
indica Burm. f., Fl. Ind.: 132, t. 39, f. 5(1768). Local name: Paniala. A spiny shrub.

\section{Caricaceae}

Carica papaya L., Sp. Pl.: 1036(1753). Local name: Pepe. An herbaceous tree with milky latex.

\section{Capparaceae}

Cleome viscosa L., Sp. Pl.: 672(1753). Local name: Hurh uria. An erect, glandularpubescent herb.

\section{Brassicaceae}

Rorippa indica (L.) Hiern., Cal. Afr. Pl. Wel. W. Pt. 1: 26(1896). Sisymbrium indicum L., Mant. 1: 93(1767). Local name: Ban Sarisha. A small herb.

\section{Myrsinaceae}

Maesa montana A. DC. in DC., Prodr. 8: 79(1844). Local name: Ramjoni. A bushy tree.

\section{Mimosaceae}

Acacia concinna (Willd.) DC. Prodr. 2: 464(1825). Mimosa concinna Willd. Sp. Pl. 4: 1039(1805). Local name: Banritha. A shrub with conical and hooked prickles.

A. auriculiformis A. Cunn. ex Benth. in London J. Bot. 1: 377 (1842). A. moniliformis Griseb. in Goett. Abh. 19:136(1874). Local name: Akashmoni. A middle sized tree.

Albizia procera (Roxb.) Benth. Journ. Bot. 3: 89(1844). Acacia procera Roxb., Pl. Corom. 2:12. t. 121(1798). Local name: Sil Koroi. A medium sized tree.

Mimosa intisia L., DC., Prod. 2: 429(1832). Local name: Bara Lajjabati. A straggling shrub.

M. pudica L., Sp. Pl.: 518(1753). Local name: Lajjabati. A prickly woody herb.

\section{Caesalpiniaceae}

Caesalpinia pulcherrima (L.) Sw. Obs.: 166(1971). Poinciana pulcherrima L., Sp. Pl. : 380(1753). Local name: Radhachura. A much branched shrub.

Cassia fistula L., Sp. Pl.: 377(1753). Local name: Sonalu. A deciduous tree.

C. occidentalis L., Sp. Pl.: 377(1753). Local name: Kalkasunda. An annual shrub.

C. tora L., Sp. Pl.: 376(1753). Local name: Chakunda. An annual shrubby herb.

Tamarindus indica L., Sp. Pl.: 34(1753). Local name: Tentul. An evergreen tree.

\section{Fabaceae}

Atylosia scarabaeoides (L.) Benth. in Miq., Pl. Jungh.: 245(1852). Dolichos scarabaeoides L., Sp. Pl.: 726(1753). Local name: Ban Kalai. A branched herb.

Butea monosperma (Lam.) Taub. in Engl. \& Prantl, Pflanzenfam. 3(3): 365(1984). Erythrina monosperma Lam., Encycl. 1: 391(1783). Local name: Palash. A deciduous tomentose tree. 
Cajanus cajan (L.) Millsp. in Field Columb. Mus. Bot. 2: 53(1900). Cytisus cajan L., Sp. Pl.: :739(1753). Local name: Arhar. An erect herb.

Centrosema pubescens Benth., Comm. Legum. Gen.: 55(1837). A climber.

Crotolaria calycina Schrank, Pl. Rar. Monac.: t. 12(1819). An erect herb.

C. pallida Aiton, Hort. Kew. 3: 20(1789). Local name: Jhanjhani. A glabrescent undershrub.

Dalbergia sissoo Roxb., [Hort. Beng.: 53(1814), nom. nud. \&] ex DC. Prodr. 2: 416(1825). Local name: Shishu. A large deciduous tree.

Desmodium gangeticum (L.) DC. Prodr. 2: 327(1825). Hedysarum gangeticum L., Sp. Pl.: 746(1753). Local name: Satpani. A suberect undershrub.

D. pulchellum (L.) Benth., Fl. Hongk.: 83(1861). Hedysarum pulchellum L., Sp. Pl.: 747(1753). Local name: Juta-Salpani. A shrub.

D. triflorum (L.) DC. Prodr. 2: 334(1825), excl. syn. cit. Hedysarum triflorum L., Sp. Pl.: 749(1753), p.p. Local name: Kulaliya. A prostrate herb.

Erythrina variegata L., in Stickm. Herb. Amboin.: 10(1754). Local name: Mandar. A deciduous prickly tree.

Flemingia strobilifera R. Br. in Ait., Hort. Kew. ed. 2(4): 350(1812). An erect shrub.

\section{Lythraceae}

Lagerstroemia speciosa Pers., Syn: 2(1937). Local name: Jarul. A large deciduous tree.

\section{Myrtaceae}

Eucalyptus citriodora Hook. in Mitch. Journ. Trop. Austral.: 235 (1848). A tall tree.

Psidium araca Raddi, Opusc. Sc. 4: 252(1823). Local name: Tok Peyara. A bushy shrub.

P. guajava L., Sp. Pl.: 470(1753). Local name: Peyara. A large shrub or small tree.

Syzygium cumini (L.) Skeels in U.S. Dept. Agr. Bull. 248: 25(1912). Myrtus cumini L., Sp. Pl.: 471(1753). Local name: Jam. A large evergreen tree.

S. fruticosum (Roxb.) DC., Prodr. 3: 260(1828). Eugenia fruticosa Roxb., Fl. Ind. ed. 2(2): 487(1832). Local name: Khudijam. A small tree.

\section{Onagraceae}

Ludwigia hyssopifolia (G. Don) Exell. Garica de Orta 5: 471(1957). Jussiaea hyssopifolia G. Don, Gen. Syst. 2: 693(1832). A branched herb.

\section{Melastomaceae}

Melastoma malabathricum L., Sp. Pl.: 390(1753). Local name: Bantezpata. A shrub.

\section{Combretaceae}

Terminalia arjuna (Roxb.) Wight \& Arn., Prodr. 314(1834). Pentaptera arjuna Roxb., [Hort. Beng. 34(1814), nom. nud.] Fl. Ind. 2: 440(1832). Local name: Arjun. A large tree. 


\section{Loranthaceae}

Dendrophthoe falcata ( Linn. f.) Etting. in Denksehr. Akad. Wissen. Math.-Naturw. 32: 52 (1872). Loranthus falcatus L. f., Suppl.: 221(1781). Local name: Bancha. A parasitic plant on Mango tree with terete branchlets.

\section{Euphorbiaceae}

Antidesma ghaesembilla Gaertn., De Fruct. 1: 189,t. 39(1788). Local name: Timtoa. A small tree.

Bridelia stipularis Bl. Bijd.: 597(1826). A shrub.

Glochidion multiloculare Muell. - Arg. in DC., Prodr. 15: 279(1866). Local name: Aniatory. A shrub.

Macaranga denticulata (Bl.) Muell. - Arg. in DC., Prodr. 15(2): 1000(1866). Mappa denticulata Bl., Bijdr.: 625 (1825). Local name: Jhakura. A small tree.

Manihot esculenta Crantz, Inst. 1: 167(1766). Local name: Kasava, Simul Alu. A subherbaceous shrub.

Phyllanthus embelica L., Sp. Pl.: 982(1753). Local name: Amloki. A medium-sized deciduous tree.

P. fraternus Webster, Contr. Gray. Herb. 176: 53(1955). Local name: Bhui amla. An erect glabrous herb.

P. reticulatus Poir., Encycl. 5: 298(1804). Local name: Chitki. A large scandent shrub.

Ricinus communis L., Sp. Pl.: 1007(1753). Local name: Redhi. An evergreen soft wooded shrub.

\section{Rhamnaceae}

Zizyphus rugosa Lam., Encycl. 3: 319(1789). Local name: Jangli Boroi. A small tree with stout thorns.

\section{Anacardiaceae}

Lannea coromandelica (Houtt.) Merr., J. Arnold. Arbor. 19: 353(1983). Dialium coromandelicum Houtt., Nat. Hist. 2: 39. t. 5. f. 2(1774). Local name: Jiga, Jeol. A middle-sized deciduous tree.

Mangifera indica L., Sp. Pl.: 200(1753). Local name: Am. A tree.

\section{Meliaceae}

Aphanamixis polystachya (Wall.) Parker in Ind. For. 57: 486(1931). Aglaia polystachya Wall. in Roxb., Fl. Ind. 2: 429(1824). Local name: Pitraj. A tree with dense spreading crown.

Azadirachta indica A. Juss. in Mem. Mus. Paris 19: 220. t. 2(1830). Local name: Nim. A large deciduous tree. 
Melia azedarach L., Sp. Pl.: 384(1753). Local name: Ghora Nim. A middle-sized deciduous tree.

Swietenia mahagoni (L.) Jacq. Enum. Pl. Carib.: 4(1760). Swietenia mahagoni L., Sp. Pl. ed. 2: 271(1762). Local name: Mehagani. A large tree.

\section{Rutaceae}

Aegle marmelos (L.) Correa in Trans. Linn. Soc. 5: 222(1800). Crataeve marmelos L., Sp. Pl.: 444(1753). Local name: Bel. A small deciduous tree with erect stout axillary thorns.

Glycosmis pentaphylla (Retz.) A. DC., Prod. 1: 538(1824). Limonia pentaphylla Retz., Obs. Bot. 5: 24(1788). Local name: Datmajan. An evergreen much-branched shrub.

Micromelum minutum (Forst.f.) Wight \& Arn., Prod. 1: 448(1834). Limonia minuta Forst. f., Prod.: 33(1786). Local name: Bankutch. A small unarmed tree.

Zanthoxylum rhetsa (Roxb.) DC., Prod.: 728(1824). Fagara rhetsa Roxb., 1. c. 437 (1820). Local name: Bajrang. An evergreen small tree.

\section{Oxalidaceae}

Oxalis corniculata L., Sp. Pl.: 435(1753). Local name: Amrul Shak. A procumbent herb with long, slender, creeping stems.

\section{Apiaceae}

Centella asiatica (L.) Urban in Mart., Fl. Bras. 11: 287(1879). Hydrocotyle asiatica L., Sp. Pl. 1: 234(1753). Local name: Thankuni.

A trailing herb.

\section{Apocynaceae}

Alstonia scholaris (L.) Brown, Mem. Wern. Nat. Hist. Soc. 1: 76(1811). Alstonia scholaris L., Mant. Pl. 1: 53(1767). Local name: Chhatim. A large deciduous tree.

Holarrhena pubescens (Buch. - Ham.) Wall. ex G. Don, Gen. Syst. 4: 78(1838). Echinites pubescens Buch. -Ham. in Trans., Linn. Soc. 13: 524(1822). Local name: Kurchi. A small tree.

Rauvolfia serpentina Benth. ex Kurz, For. Fl. Brit. Burma 2: 171(1877). Local name: Sarpagandha. A woody herb.

\section{Asclepiadaceae}

Calotropis gigantea (L.) Dryand. in Aiton, Hort. Kew. ed. 2(2): 78(1811). Asclepias gigantea L., Sp. Pl.: 214(1753). Local name: Akanda. A large shrub with milky juice.

Hemidesmus indicus (L.) R. Br. in Aiton, Hort. Kew. ed. 2( 2): 75(1811). Periploca indica L., Sp. Pl.: 211(1753). Local name: Annantamul. A prostrate or slightly twining undershrub with aromatic roots. 


\section{Solanaceae}

Solanum lasiocarpum Dunal, Hist. Solanum: 222 (1813) Solanum indicum L., Sp. Pl.: 187(1753). Local name: Gurkamai. A much branched undershrub.

S. nigrum L., Sp. Pl.: 186(1753). Local name: Puti Begun. A herb.

S. torvum Sw., Nov. Gen. Sp. Pl.: 47(1788). Local name: Gota Begun. A shrub.

\section{Convolvulaceae}

Ipomoea fistulosa Mart. ex Choisy in DC., Prodr. 9: 349(1845). Local name: Dholkalmi. A shrub containing milky juice.

Merremia umbellata (L.) Hallier. f., Bot. Jahrb. 16: 552(1893). Convolvulus umbellatus L., Sp. Pl. : 155(1753). Local name: Sada kalmi. A herbaceous twiner.

\section{Cuscutaceae}

Cuscuta reflexa Roxb., Pl. Corom. 2: 3, t. 104(1798). Local name: Swarnalata. A twining parasitic herb.

\section{Boraginaceae}

Heliotropium indicum L., Sp. Pl. : 130(1753). Local name: Hatisur. An erect herb.

\section{Verbenaceae}

Clerodendrum viscosum Vent., Jard. Malm. 1: t. 25(1803). Local name: Bhant. A softy tomentose woody herb.

Gmelina arborea Roxb., Fl. Ind. 3: 84 (1832). Local name: Gamari. A deciduous unarmed tree.

Lantana camara L., Sp. Pl. ed. 1, 2: 627(1753) var. camara Schau in Mart., Fl. Bras., 9: 256(1851). A branching shrub.

Premna bengalensis Clarke in Hook. f., Fl. Brit. Ind. 4: 577(1882). Local name: Dauli. A tree.

Vitex peduncularis Wall. Cat.: 1753(1825). Local name: Horina. A middle-sized tree.

\section{Lamiaceae}

Gomphostemma parviflorum Wall. [Cat. 60, n. 215(1830): nom. nud]. Local name: Jateribormala. A perennial herb.

Hyptis suaveolens Poit, Ann. Mus. Natl. Hist. Nat. 7: 472 t. 29, f. 2(1806). Local name: Tokma. An aromatic herb.

Leucas aspera Spreng. Syst. 2: 743(1825). Local name: Swetadrone, Dandakalos. A herb.

Ocimum sanctum Linn., Mart. 1: 85(1767). Local name: Tulsi. A much branched, softly hairy herb. 


\section{Scrophulariaceae}

Scoparia dulcis L., Sp. Pl.: 116(1753). Local name: Bandhoney.

An erect or ascending herb.

Torenia vagans Roxb., Fl. Ind. 3: 96(1832).

A herb.

\section{Acanthaceae}

Justicia gendarussa Burm. f., Fl. Ind.: 10(1768). Local name: Jagatmadan. An undershrub.

Lepidagathis incurva F. Ham. ex D. Don. Prodr.: 119(1825). A herb.

Nelsonia canescens (Lam.) Spreng. in L., Sys. Veg. ed. 16, 1: 42(1824). Justicia canescens Lam., Tab. Encycl. Method. Bot. 1: 40(1791). Local name: Paramul. A trailing herb.

Phaulopsis dorsiflora (Retz.) Sant. in Kew Bull. 1948: 276(1948). Ruellia dorsiflora Retz., Obs. 6: 31(1791). A much branched hairy herb.

Rungia pectinata (L.) Nees in DC., Prodr. 11: 469(1847). Justicia pectinata L. Amoen. Acad. 4: 293(1759). Local name: Pindi. A much branched suberect herb.

Strobilanthes scaber Nees in Wall., Pl. As. Rar. 3: 84(1832). A herb.

\section{Rubiaceae}

Borreria articularis (Linn.f.) Williams, Bull. Herb. Boissier (Ser.) 2, 5: 956(1905). Spermacoce articularis Linn. f., Suppl.: 119(1781). Local name: Madnabata kadu. A procumbent rough hispid herb.

Hedyotis scandens Roxb., Hort. Beng.: 10(1814), nom. nud. \& Fl. Ind. 1: 364(1820). Local name: Lataguji. A climber.

Ixora cunifolia Roxb., Fl. Ind. 1: 385(1820). Local name: Beophul. A shrub.

Mussaenda roxburghii Hook. f., Fl. Brit. Ind. 3: 87(1880). Local name: Silchaonri. An erect large shrub.

\section{Asteraceae}

Ageratum conyzoides L., Sp. Pl.: 839(1753). Local name: Fulkuri. A herb.

Blumea lacera (Burm. f.) DC. in Wight., Clarke, Comp. Ind.: 76(1876). Conyza lacera Burm. f., Fl. Ind.: 180, t. 59. f. 1(1786). Local name: Barakukshima. An erect aromatic herb.

Elephantopus scaber L., Sp. Pl.: 814(1753). Local name: Gejiashak. An erect herb with creeping rootstock..

Eupatorium odoratum Linn., Syst. Nat. ed. 10: 1205(1759). Local name: Germanlata, Assamlata. A herb. 
Gnaphalium indicum auct. non L., C. B. Clarke, Comp. Ind.: 114(1876). Local name: Bara Kamra. A wooly herb.

Mikania cordata (Burm.) Robinson, Contr. Gray herb. 104: 65(1934). Eupatorium cordatum Burm., Fl. Ind.: 176, t. 58, fig. 2(1768). Local name: Refugee lata. A herb.

Spilanthes calva DC. in Wight, Contrib.: 19(1834). Local name: Marhatitiga. An annual herb.

Synedrella nodiflora (L.) Gaertn., Fruct. 2: 456. t. 171. f. 7(1791). Verbesina nodiflora L., Amoen. Acad. 4: 290(1759). An erect branching annual herb.

Vernonia cineria (L.) Lees., Linnaea 4(1): 291(1829). Conyza cinerea L., Sp. Pl.: 862(1753). Local name: Kuksim. An erect herb.

\section{LILIOPSIDA (MONOCOTS)}

\section{Arecaceae}

Borassus flabellifer L., Sp. Pl.: 1187(1753). Local name: Tal. A tall palm.

Cocos nucifera L., Sp. Pl.: 1188(1753). Local name: Narikel. A coconut palm.

Phoenix sylvestris (L.) Roxb., Fl. Ind. 3: 787(1832). Elate sylvestris L., Sp. Pl.: 1189(1753). Local name: Khejur. A tall palm.

\section{Cyperaceae}

Cyperus iria L., Sp. Pl. ed. 1: 45(1753). Local name: Barachancha. An annual herb.

C. rotundus L., Sp. Pl.: 45(1753). Local name: Motha ghas. A perennial grass.

Fimbristylis miliacea (L.) Vahl, Enum. Pl. 2: 287(1806). Scirpus miliaceus L., Syst. ed. 10: 868(1759). Local name: Bara javani. A perennial, tufted herb.

Kyllinga monocephala Rottboel., Descr. Ic. Rar. Nov. Pl.: 13, t. 4, f. 4(1773). Local name: Nirbirshaghas. A perennial herb, rhizome covered with scale.

Mariscus sumatrensis (Retz.) Raynal, Adansonia 15: 110(1975). Kyllinga sumatrensis Retz., Obs. Bot. 4: 13(1786). Local name: Bara guthubi. A perennial herb, stoloniferous in some cases.

Scleria levis Retz., Obs.: 4(1786). A perennial herb with short rhizome.

\section{Poaceae}

Bambusa balcooa Roxb., Fl. Ind. 1: 196(1820). Local name: Barakbans. A tall stout, densely caespitose bamboo.

Cynodon dactylon (L.) Pers. Syn. Pl. 1: 85(1805). Panicum dactylon L., Sp. Pl.: 58(1753). Local name: Durbaghas. A perennial grass.

Eleusine indica (Linn.) Gaertn., Fruct. 1: 8(1789). Cynosursus indicus Linn., Sp. Pl.: 72(1753). Local name: Malankuri. A tufted annual grass. 
Eragrostis cilianensis (All) Janchen, Mitt. Naturw. Univ. Wien 5 (9): 110 (1907). E. major Host. Gram. Austr. 4: 14. t. 24 (1809). An annual grass.

E. unioloides (Retz.) Nees ex Steud., Syn. Pl. Glum. 1: 264(1854). Poa unioloides Retz., Obs. Bot. 5: 19(1789). An annual grass.

Melocanna bambusoides Trin. in Spreng., Neue Enterdeck 2: 45(1821). An evergreen unarmed bamboo.

Panicum brevifolium L., Sp. Pl.: 59(1753). A slender decumbent grass.

P. notatum Retz., Obs. Bot. 4: 18(1786). A tufted perennial grass.

P. paludosum Roxb., Fl. Ind. 1: 310(1820). Local name: Barati. A perennial grass.

P. repens L., Sp. Pl. ed. 2: 87(1762). Local name: Dhanighas. A perennial grass.

Paspalidium flavidum (Retz.) A. Camus in Lecomte, Fl. Gen. de I’Indo-Chine 7: 419(1922). Panicum flavidum Retz., Obs. Bot. 4: 15(1876). An annual grass.

Setaria glauca (Linn.) P. Beauv., Ess. Agrost.: 51, 169, 178(1812). Panicum glaucum Linn., Sp. Pl. ed. 1: 56(1753). A tufted annual grass.

\section{Zingiberaceae}

Curcuma zedoaria (Christm.) Roscoe., Trans. Linn. Soc. London 8: 354(1807). Amomum zedoaria Christm. in Christm. \& Panzer, Linn. Pflanzensyst. 5: 12(1779). Local name: Shoti. A stemless herb with pale yellow-white rhizome.

\section{Costaceae}

Costus speciosus (Koenig) Smith, Trans. Linn. Soc. London 1: 249(1791). Banksea speciosa Koenig in Retz., Obs. Bot. 3: 75(1783). A herb, 2-3m long.

\section{Smilacaceae}

Smilax zeylanica L., Sp. Pl.: 1029(1753). Local name: Kumari lata. A large prickly climber.

\section{References}

Brandis, D. 1906. Indian Trees (2nd Repr. 1978). Bishen Singh Mahendra Pal Singh, Dehra Dun, 767 pp.

Cronquist, A. 1981. An integrated system of classification of flowering plants. Columbia University Press, New York, 1262 pp.

Deb, D.B. 1981. The Flora of Tripura State 1 : 1-50. R.K. Jain, Today \& Tomorrow's Printers and Publishers, New Delhi.

Deb, D.B. 1983. The Flora of Tripura State 2 : 1-601. R.K. Jain, Today \& Tomorrow's Printers and Publishers, New Delhi.

Hossen, M.M. and Dewan, T.A. 2004. Moinamoti-Lalmai. Archeological Division, Ministry of Culture and Heritage. $60 \mathrm{pp}$.

Hooker, J.D. 1872-1897. The Flora of British India Vols. 1-7 (Repr. 1973). Bishen Singh Mahendra Pal Singh, Dehra Dun. 
Kanjilal, U.N., Das, A., Kanjilal, P.C. and De, R.N. 1939. Flora of Assam 3 : 1-578 (Ind. Repr. 1982). A Von Book Company, Delhi.

Kanjilal, U.N., Kanjilal, P.C. and Das, A. 1934. Flora of Assam 1 : 1-386 (Ind. Repr. 1982). A Von Book Company, Delhi.

Kanjilal, U.N., Kanjilal, P.C. and Das, A. 1938. Flora of Assam 2 : 1-409 (Ind. Repr. 1982). A Von Book Company, Delhi.

Kanjilal, U.N., Kanjilal, P.C., De, R. N. and Das, A. 1940. Flora of Assam 4 : 1-377 (Ind. Repr. 1982). A Von Book Company, Delhi.

Khan, M. S. 1977. Onagraceae. In: Khan, M. S. (ed.). Flora of Bangladesh. Fasc. 6 : 1-10. Bangladesh National Herbarium and Bangladesh Agricultural Research Council, Dhaka.

Khan, M. S. 1984. Dipterocarpaceae. In: Khan, M. S. (ed.). Flora of Bangladesh. Fasc. 25 : 1-15. Bangladesh National Herbarium and Bangladesh Agricultural Research Council, Dhaka.

Khan, M. S. 1985. Convolvulaceae. In: Khan, M. S. (ed.). Flora of Bangladesh. Fasc. 30 : 1-59. Bangladesh National Herbarium and Bangladesh Agricultural Research Council, Dhaka.

Matthew, K. M. 1999. The flora of the Palni Hills, South India 1 : 1-575. The Rapinat Herbarium Tiruchirapalli, India.

Matthew, K. M. 1999a. The flora of the Palni Hills, South India 2 : 576-1196. The Rapinat Herbarium Tiruchirapalli, India.

Matthew, K. M. 1999b. The flora of the Palni Hills, South India 3 : 1197-1880. The Rapinat Herbarium Tiruchirapalli, India.

Prain, D. 1903. Bengal Plants 1: 1-487 (Ind. Repr. 1963). Botanical Survey of India, Calcutta.

Prain, D. 1903a. Bengal Plants 2: 488-1013 (Ind. Repr. 1963). Botanical Survey of India, Calcutta. 